\title{
Homodyne en face optical coherence tomography
}

\author{
Zahid Yaqoob \\ Department of Electrical Engineering, MC 136-93, California Institute of Technology, Pasadena, California 91125 \\ Jeff Fingler \\ Department of Applied Physics, MC 139-74, California Institute of Technology, Pasadena, California 91125
}

Xin Heng and Changhuei Yang

Department of Electrical Engineering, MC 136-93, California Institute of Technology, Pasadena, California 91125

\begin{abstract}
Received January 10, 2006; revised March 30, 2006; accepted April 7, 2006; posted April 17, 2006 (Doc. ID 67156) We demonstrate, for what we believe to be the first time, the use of a $3 \times 3$ fiber-optic coupler to realize a homodyne optical coherence tomography (OCT) system for en face imaging of highly scattering tissues and turbid media. The homodyne OCT setup exploits the inherent phase shifts between different output ports of a $3 \times 3$ fiber-optic coupler to extract amplitude information of a sample. Our homodyne en face OCT system features a measured resolution of $14 \mu \mathrm{m}$ axially and $9.4 \mu \mathrm{m}$ laterally with a $90 \mathrm{~dB}$ signal-to-noise ratio at $10 \mu$ s integration time. En face OCT imaging of a stage 52 Xenopus laevis was successfully demonstrated at a depth of $600 \mu \mathrm{m}$ within the sample. (C) 2006 Optical Society of America
\end{abstract}

OCIS codes: $170.0180,170.4500,170.3880$.

Optical coherence tomography (OCT) is a noninvasive technique for high-resolution depth-resolved imaging of highly scattering biological samples. ${ }^{1}$ En face OCT systems ${ }^{2,3}$ belong to a special class of OCT that preferentially provides sample images in a plane normal to the optical axis. The reported en face OCT systems can be categorized into two types: full-field OCT designs ${ }^{3}$ and two-dimensional (2D) beam scanning designs. ${ }^{2,4}$ Most of the reported en face OCT systems use heterodyne techniques to measure the interference signal. ${ }^{2-4}$ The use of a heterodyne carrier frequency requires a minimum dwell time (equal to one full oscillation period) for each pixel acquisition. We note that a homodyne-detection-based en face OCT system can be simpler to implement and does not impose a dwell time requirement. Beaurepaire $e t$ $a l .{ }^{5}$ reported such a system. However, in that system the amplitude and phase information are entangled. In this Letter we present a $3 \times 3$ fiber-optic-couplerbased homodyne OCT system that is capable of obtaining the amplitude independent of the phase information. This en face OCT system exploits the nontrivial phase shift in a $3 \times 3$ fiber-optic coupler, which permits simultaneous and independent acquisition of the sample phase and amplitude information. ${ }^{6}$ The proposed design is simple, easy to implement, and offers a low-cost and high-speed en face imaging solution.

Figure 1(a) shows the design of our $3 \times 3$ fiber-optic coupler-based homodyne en face OCT system. A broadband light source (SuperlumDiodes Ltd., D1300-HP) of $\lambda_{0}=1300 \mathrm{~nm}, \Delta \lambda \sim 85 \mathrm{~nm}$ is coupled to a $3 \times 3$ coupler (AC Photonics) via a $2 \times 2$ coupler $(\mathrm{AC}$ Photonics). A 2D mirror scanner (Physik Instrumente, S-334.2SL) in the sample arm is used to scan the sample beam. A $4 f$ lens system $(f=150 \mathrm{~mm})$ along with a $20 \times$ microscope objective (Olympus, LMPLAN IR) helps to achieve linear scanning across the sample. Light returning from the sample and reference arms is collected by three detectors (New Focus Model 2011) marked $\mathrm{D}_{1}, \mathrm{D}_{2}$, and $\mathrm{D}_{3}$. A similar detector $\left(\mathrm{D}_{4}\right)$ is used to measure source intensity; the measurement can then be used to correct for the source fluctuations. The data are acquired by using a 16-bit analog-to-digital converter (National Instruments, PXI-6120).

To facilitate our explanation of the homodyne OCT concept, we first define a set of coefficients $\alpha_{m n}$, where $\alpha_{m n}$ denotes the power transfer coefficient from port $m$ to port $n$ of the $3 \times 3$ coupler. By the na-

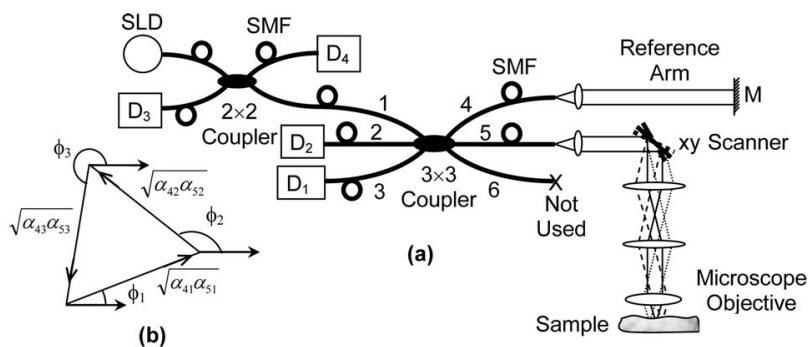

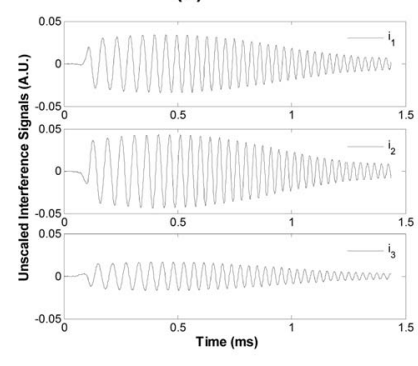

(c)

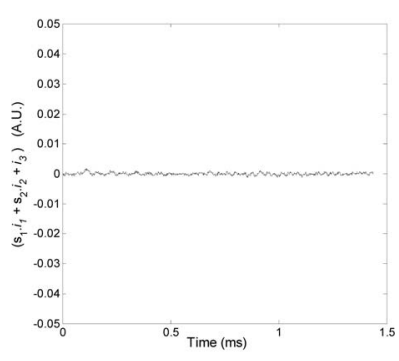

(d)
Fig. 1. (a) Schematic of homodyne en face OCT system. (b) Schematically demonstrates triangular relationship between $3 \times 3$ coupler coefficients and interferometric phase shifts between the coupler arms. (c) Unscaled interference signals at detectors $\mathrm{D}_{j}$ after dc removal. (d) Sum of the scaled interference signals. SLD, superluminescent diode; $\mathrm{SMF}$, single-mode fiber; $i_{j}$, $j$ th interference signal. 
ture of the fiber coupler, reciprocity $\left(\alpha_{m n}=\alpha_{n m}\right)$ applies. The optical signal detected by the $j$ th detector can be expressed as

$$
\begin{aligned}
P_{j}(z)= & P_{r, j}+P_{s, j}+2\left(1 / s_{j}\right) \sqrt{\alpha_{41} \alpha_{4 j} \alpha_{51} \alpha_{5 j}} \sqrt{P_{r}} \\
& \times\left\{\sqrt{P_{s}(z)} \otimes \gamma(z)\right\} \cos \left[2 k_{0} z+\psi(z)+\phi_{j}\right],
\end{aligned}
$$

where $P_{r, j}$ and $P_{s, j}$ represent the total measured power from the reference and sample arms, respectively; $1 / s_{j}$ is the loss scale factor that accounts for coupler loss and detector loss to the detected interferometric signal. The factors $P_{r}, P_{s}(z)$, and $\gamma(z)$ are the returning reference power, the returning coherent light from depth $z$ in the sample, and the autocorrelation function of the broadband light source, respectively. $k_{0}$ is the optical wavenumber (corresponding to the center wavelength) in air, and $\psi(z)$ is the intrinsic reflection phase shift of the sample at depth $z$. The interferometric phase shift $\phi_{j}$ differences are dependent on the relative values of $\alpha_{m n}$; for example, if $\alpha_{m n}$ are all equal (ideal equalsplit coupler), the two $\phi_{j}$ should differ from each other by $120^{\circ}$. The method of extracting complex interferometric signal components from such homodyne measurements works as long as none of the differences between $\phi_{j}$ are equal to $0^{\circ}$ or $180^{\circ} .{ }^{6}$

We define $i_{j}$ as the interferometric portion of $P_{j}(z)$. Assigning $s_{1} i_{1}$ as the real part of the complex interferometric signal (set $\phi_{1}=0$ ), the real and imaginary parts of the interference signal can then be written as ${ }^{6}$

$$
\begin{aligned}
i_{\mathrm{Re}}= & s_{1} i_{1}=2 \sqrt{\alpha_{41} \alpha_{41} \alpha_{51} \alpha_{51}} \sqrt{P_{r}}\left\{\sqrt{P_{s}(z)} \otimes \gamma(z)\right\} \\
& \times \cos \left[2 k_{0} z+\psi(z)\right],
\end{aligned}
$$

$$
\begin{aligned}
i_{\mathrm{Im}}= & 2 \sqrt{\alpha_{41} \alpha_{41} \alpha_{51} \alpha_{51}} \sqrt{P_{r}}\left\{\sqrt{P_{s}(z)} \otimes \gamma(z)\right\} \\
& \times \sin \left[2 k_{0} z+\psi(z)\right], \\
= & \frac{s_{1} i_{1} \cos \left(\phi_{2}\right)-s_{2} i_{2} \beta}{\sin \left(\phi_{2}\right)}, \quad \text { where } \beta=\sqrt{\frac{\alpha_{41} \alpha_{51}}{\alpha_{42} \alpha_{52}}} .
\end{aligned}
$$

The derivation of Eq. (2b) is detailed in Ref. 6. Complete quadrature information (amplitude and phase) can be simultaneously obtained as

$$
\begin{aligned}
i_{0} & =\sqrt{i_{\mathrm{Re}}^{2}+i_{\mathrm{Im}}^{2}}, \\
2 k_{0} z+\psi(z) & =\tan ^{-1}\left(i_{\mathrm{Im}} / i_{\mathrm{Re}}\right) .
\end{aligned}
$$

To use Eqs. (2) and (3), we have to experimentally quantify the coefficients $\beta, \phi_{j}$, and $s_{j}$. As we are primarily interested in the relative magnitudes of $s_{j}$, we can make a simplifying assumption that $s_{3}=1$. We quantify all of these parameters through a calibration process in which we replace the sample with a mirror. A slight tilt in the sample mirror results in phase modulation in our detected signals as the sample beam scans across the mirror. At each detector, two sets of 3000 sample points were acquired at $80 \mathrm{kHz}$ with the reference arm blocked (to determine
$\left.P_{s, j}\right)$ and unblocked. A knife-edge introduced into the sample arm blocks the sample light for a fraction of each scan, allowing $P_{r, j}$ to be measured. The two data sets were offset balanced and subtracted to determine the interference signals at the three detectors [see Fig. 1(c)]. The three interferometric signals should add up to zero by the law of power conservation. ${ }^{6}$ This condition enables us to determine the scaling factors $s_{1}$ and $s_{2}$ in our experimental situation. Specifically, we can write a matrix

$$
\left[\begin{array}{ll}
i_{1}\left(t_{1}\right) & i_{2}\left(t_{1}\right) \\
i_{1}\left(t_{2}\right) & i_{2}\left(t_{2}\right)
\end{array}\right]\left[\begin{array}{l}
s_{1} \\
s_{2}
\end{array}\right]=-\left[\begin{array}{l}
i_{3}\left(t_{1}\right) \\
i_{3}\left(t_{2}\right)
\end{array}\right],
$$

where $i_{j}\left(t_{1}\right)$ and $i_{j}\left(t_{2}\right)$ are the three interferometric signal values at two different time instances. We find the best fit values for factors $s_{1}$ and $s_{2}$ by inserting multiple sets of interferometric data points into Eq. (4). Figure 1(d) shows a representative plot of the sum of the scaled interference signals. Knowing the value of each $s_{j}$, the values of the peak fringe height from each scaled interferometric signal, $s_{j} i_{j}$, which are proportional to $\sqrt{\alpha_{4 j} \alpha_{5 j}}$ [see Fig. 1(b)], can then be used to determine $\beta$ as well as $\left(\phi_{j}-\phi_{k}\right)$. Four sets of coupler characterizations were performed at different times, which yielded an averaged $\beta$ of $0.95 \pm 0.01$ and the interferometric phase shifts $\left(\phi_{j}-\phi_{k}\right)$ at $122.7^{\circ} \pm 0.2^{\circ}, 121.1^{\circ} \pm 0.8^{\circ}$, and $116.2^{\circ} \pm 0.7^{\circ}$, respectively.

To construct the homodyne OCT signal, the reference arm dc power $P_{r, j}$ is first subtracted from the measured detector power $P_{j}(z)$. Next, the summation $\sum_{k=1}^{3} s_{k}\left[P_{k}(z)-P_{r, k}\right]$, with $s_{3}$ set as 1 , removes all the interference terms, leaving only the sum of the scaled contributions from the sample dc power of all the detectors. Assuming that the instantaneous ratio of the measured sample power of the detectors is equal to the average ratio of the measured sample power of each detector, the instantaneous scaled sample power measured in each detector is determined by

$$
s_{j} P_{s, j}=\frac{\sum_{k} s_{k}\left[P_{k}(z)-P_{r, k}\right]}{\sum_{k}\left(\left\langle P_{s, k}\right\rangle /\left\langle P_{s, 2}\right\rangle\right)} \frac{\left\langle P_{s, j}\right\rangle}{\left\langle P_{s, 2}\right\rangle} .
$$

Removing the values of Eq. (5) from the scaled reference-removed measurements, the remaining terms are the scaled interference signals $s_{j} i_{j}$. The OCT signal can then be determined by using Eq. (3a).

Our proof-of-concept homodyne en face OCT system features measured axial and lateral resolutions of $14 \mu \mathrm{m}$ (theoretical $8.7 \mu \mathrm{m}$ ) and $9.4 \mu \mathrm{m}$ (theoretical $3.2 \mu \mathrm{m}$ ), respectively. The discrepancy between the measured and theoretical axial resolution can be attributed in part to the spectral variations in the power transfer coefficients of the $3 \times 3$ coupler, which we have assumed to be spectrally flat. The confocal parameter was measured as $97 \mu \mathrm{m}$. The signal-tonoise ratio (SNR) of the homodyne en face OCT is measured as $90 \mathrm{~dB}$ for a $10 \mu \mathrm{s}$ integration time (the corresponding theoretical shot-noise-limited SNR is $\sim 102 \mathrm{~dB}$ ). Figure 2 shows the theoretical (shot-noise- 


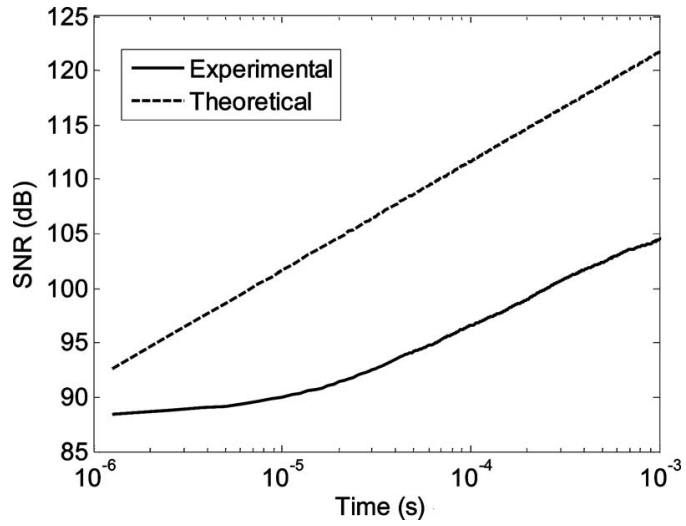

Fig. 2. Plots of theoretical (shot-noise-limited) as well as measured SNR versus integration time of the homodyne en face OCT system.

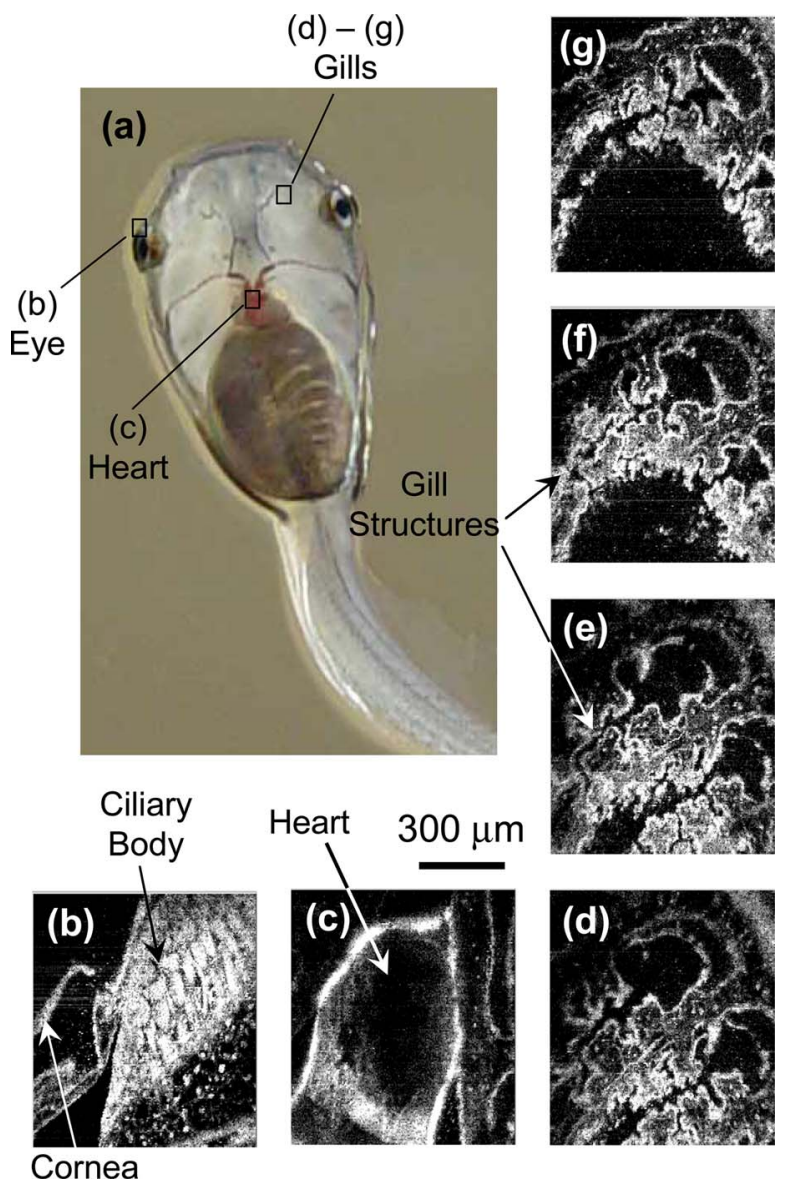

Fig. 3. (Color online) (a) Photograph of a stage 52 Xenopus leavis showing locations where en face images were acquired by using the homodyne OCT setup. Shown are the en face OCT images of (b) cornea and ciliary body in the eye, (c) heart, and (d) gill structures at a depth of $600 \mu \mathrm{m}$ into the sample. (e)-(g) En face images of the gill region of (d) at $40 \mu \mathrm{m}$ depth intervals into the sample. Each en face OCT image is $\sim 790 \mu \mathrm{m} \times 900 \mu \mathrm{m}$.

limited) as well as the measured SNR plots versus the integration time for our homodyne OCT system. The integration time is varied by simply accumulating the signal for the requisite time period. The dif- ference in the SNR plots may be attributed to $3 \times 3$ coupler losses, signal loss due to the polarization mismatch between the sample and reference arms, and the remaining DC signal caused by variations in the interferometer coupling and the $3 \times 3$ coupler parameters over time because of temperature fluctuations. The effect of $1 / f$ noise on the SNR is unclear at present and deserves further study.

We also demonstrate successful homodyne en face OCT imaging in a euthanized Xenopus laevis (stage 52 ) at a $600 \mu \mathrm{m}$ depth inside the sample. Figure 3(a) is a photograph of the tadpole, indicating locations where en face OCT images were acquired. Figures 3(b)-3(d) show en face OCT images of tadpole heart as well as structures in the eye and the gill region. Figures $3(\mathrm{e})-3(\mathrm{~g})$ show en face OCT images of the gill region [shown in Fig. 3(d)] at $40 \mu \mathrm{m}$ depth intervals into the sample. The size of each en face OCT image is $\sim 790 \mu \mathrm{m} \times 900 \mu \mathrm{m}$. The data were acquired at $80 \mathrm{kHz}$, with a line scan rate of $\sim 23 \mathrm{~Hz}$. The data acquisition time per en face image was $\sim 9 \mathrm{~s}$. The measured SNR of the system at the image acquisition rate $(80 \mathrm{kHz})$ is $\sim 90 \mathrm{~dB}$.

In conclusion, we have proposed and experimentally demonstrated, for the first time to our knowledge, a homodyne en face OCT system based on a 3 $\times 3$ fiber-optic coupler. The system design is simple, easy to implement, highly sensitive, and allows for high-speed en face OCT imaging of highly scattering tissues and turbid media. Applications that can benefit from this simple imaging system include en face imaging of epithelial tissue layers of free surfaces of body for early detection and staging of near-surface microscopic precancerous lesions. We note that the proposed en face homodyne OCT system can be very easily integrated into a standard confocal microscope, hence leading to homodyne optical coherence microscopy that will benefit from all the advantages of homodyne detection (ease of implementation) and confocal microscopy (superior axial gating) as well as low coherence interferometry ${ }^{7}$ (high SNR as well as better depth penetration by virtue of sharper coherence gate rejection).

Z. Yaqoob's e-mail address is zyaqoob@caltech.edu.

\section{References}

1. D. Huang, E. A. Swanson, C. P. Lin, J. S. Schuman, W. G. Stinson, W. Chang, M. R. Hee, T. Flotte, K. Gregory, C. A. Puliafito, and J. G. Fujimoto, Science 254, 1178 (1991).

2. J. A. Izatt, M. R. Hee, G. M. Owen, E. A. Swanson, and J. G. Fujimoto, Opt. Lett. 19, 590 (1994).

3. A. Dubois, L. Vabre, A. C. Boccara, and E. Beaurepaire, Appl. Opt. 41, 805 (2002).

4. C. K. Hitzenberger, P. Trost, P. W. Lo, and Q. Y. Zhou, Opt. Express 11, 2753 (2003).

5. E. Beaurepaire, L. Moreaux, F. Amblard, and J. Mertz, Opt. Lett. 24, 969 (1999).

6. M. A. Choma, C. H. Yang, and J. A. Izatt, Opt. Lett. 28, $2162(2003)$.

7. R. C. Youngquist, S. Carr, and D. E. N. Davies, Opt. Lett. 12, 944 (1987). 